\title{
Reinventar el Gobierno: problemas y perspectivas $^{1}$
}

\section{Joel D. Aberbach * y Bert A. Rockman **}

\section{Introducción}

La versión americana de la Nueva Gestión Pública (New Public Management - NPM) se ha denominado comúnmente reinventar el gobierno. Su manifestación más destacada en los Estados Unidos es el Análisis de la Actuación Nacional (National Performance Review - NPR) de Al Gore, rebautizada ahora oficialmente como la Asociación Nacional para la Reinvención del Gobierno (National Partnership for Reinventing Government), pero a la que todavía frecuentemente se alude como NPR o simplemente reinvención. Gore quiere un gobiemo que -funcione mejor y cueste menos. ${ }^{2}$. Se discute poco sobre tan admirable objetivo, pero se plantean muchas cuestiones respecto de los medios para lograrlo.

Este artículo expone el núcleo del argumento de la reinvención en los Estados Unidos, lo relaciona con movimientos similares que están en marcha en muchas naciones del mundo y examina los problemas y perspectivas de la reinvención. También analiza la experiencia a la luz de una abundante legislación relacionada con la reinvención - la Ley de Actuación y Resultados del Gobierno (Government Performance and Results Act-GPRA) de 1993. Finalmente, plantea algunas cuestiones más. amplias relativas a lo que se puede y no se puede esperar que la reinvención consiga.

Una cuestión importante del artículo es que las opciones políticas se toman bajo el disfraz de reformas administrativas. Mientras que, hasta la fecha, la iniciativa de la reinvención ha enfatizado las técnicas de gestión, la cuestión fundamental es lo que los ciudadanos de un país quieren que el sector público haga. Sólo entonces cobran relevancia las técnicas de gestión.

\section{Orígenes y elementos de la reinvención}

El Análisis de la Actuación Nacional (NPR) comenzó desde el principio de la administración Clinton cuando el Presidente pidió al Vicepresidente Gore que llevase a cabo una gran revisión del gobiemo federal. Un equipo de aproximadamente 250 empleados del gobierno realizó el análisis y elaboró unos documentos modelo y un informe sumario que se ha convertido en objeto de interés y controversia entre los empleados del gobierno y los académicos. La primera fase del NPR puso énfasis en cómo trabaja el gobiemo, seguida de una segunda fase centrada más en lo que el gobiemo hace. El análisis se encuentra ahora en su tercera fase. Se ha ido centrando cada vez más en los resultados y en las agencias de :alto impacto", tales como el Servicio de Ingresos Interno (Internal Revenue Senvice) donde el fracaso spodría resultar peligroso a nivel político y de gestión. para la administración y, sin que sea casual, para las perspectivas políticas del Vicepresidente. La tercera fase de la reinvención pone énfasis en su vinculación con los objetivos de la Ley de Actuación y Resultados del Gobiemo (GPRA), que exige que las agencias especifiquen objetivos claros y que midan después si se han logrado o no los objetivos ${ }^{3}$. 
NPR es parte del movimiento mundial conocido como Nueva Gestión Pública (New Public Management - NPM). Existen algunas variantes de la Nueva Gestión Pública, pero una noción clave se ha puesto de manifiesto abiertamente ya desde el comienzo en el informe NPR en una sección titulada $\cdot$ El Problema de Raíz: las Burocracias de la Era Industrial en la Era de la Información: El informe afirma:

¿Es el gobierno intrínsecamente incompetente? Desde luego que no. ¿Están las agencias federales llenas de gente incompetente? No. El problema es mucho más profundo: Washington está lleno de organizaciones diseñadas para un entorno que ya no existe...

Desde los años 30 hasta los 60 , construimos enormes burocracias centralizadas, de arriba abajo, para que se ocuparan de los asuntos públicos. Se diseñaron después de las estructuras corporativas de la época ... Con su rígida preocupación por los procedimientos operativos estandarizados, con sus cadenas de mando verticales y con sus servicios estandarizados, estas burocracias eman fijas, pero lentas y engorrosas. $Y$ en el mundo actual de rápidos cambios, de tecnologías que brindan información rápida, de dura competición global y clientes exigentes, las burocracias grandes y verticales - públicas o privadas- no funcionan muy bien. Saturn no se dirige de la misma forma en que lo fue General Motors. Intel no se dirige en la forma en que lo fue IBM ${ }^{4}$.

El Presidente Clinton lo señaló de forma más terminante cuando anunció el establecimiento del NPR: "Brevemente, es hora de que nuestra administración se ajuste al mundo real, se apriete el cinturón, gestione sus asuntos en el contexto de una economia que se basa en la información, que cambia rápidamente y que premia la celeridad, el funcionamiento y el servicio, no las normas y las regulaciones";

En resumen, la Nueva Gestión Pública se asienta en la presunción de que las burocracias administrativas (y también muchas políticas gubernamentales) necesitan adaptarse a un mundo que ha variado. El objetivo es cambiar la administración y la cultura administrativa centrándose en los resultados organizacionales, la gestión individual y los incentivos del mercado (incluyendo, cuando sea posible, la privatización). Las estructuras y culturas burocráticas del pasado regidas por reglas serán sustituidas por organizaciones más pequeñas, flexibles y orientadas al cliente. Los nuevos sistemas de gestión son la clave. Dar a los gestores los incentivos adecuados y darles la libertad para gestionar libres de trabas en un entorno altamente competitivo y guiado por la actuación, sostienen los partidarios del NPM, produciría unos resultados que devolverán la confianza en el gobierno e incrementarán la productividad de cada dólar destinado a impuestos. El objetivo es un Estado mínimo que desarrolle sólo las funciones que son necesarias de una forma eficaz y efectiva.
Una cosa es hablar de reinventar tan venerables instituciones como las burocracias ede la era industrial. y otra cosa es hacerlo. Para sorpresa de muchos, las naciones como Gran Bretaña, Nueva Zelanda, Suecia y Australia, por nombrar unas cuantas que han tenido grandes Estados", han comenzado precisamente a hacer eso, y el staff del NPR era muy consciente de sus actividades ${ }^{6}$. La OCDE ha dedicado una página web a este tema? . El Banco Mundial predica este evangelio. Es un movimiento hecho y derecho.

Una pregunta obvia es por qué. Otra es por qué ahora. Éstas no son cuestiones fáciles de responder. La respuesta del NPR, como acabamos de ver, es que el movimiento se desarrolló porque ulas necesidades de las sociedades de la era de la información estaban colisionando con los límites de los gobiernos de la era industria ${ }^{8}$. Para resumir el argumento, las naciones, con independencia de las ideologías de aquéllos que las dirigen, se han visto empujadas a reinventar por necesidad.

Pero ¿qué es lo que hace precisamente al NPM una necesidad? Christopher Hood, un relevante analista británico, exploró un conjunto de explicaciones que competían entre sí y dio, de una forma un tanto escéptica, una respuesta no muy diferente de la del informe Gore. Considera el NPM como un reflejo administrativo de ese conjunto más amplio de cambios sociales desencadenados por el 'post-industrialismo'." Los cambios en la tecnología, concretamente en la introducción de los potentes ordenadores en red, permiten presumiblemente a los gobiernos subcontratar más de lo que lo hacían antes y dirigir organizaciones más flexibles y planas. Y los cambios en las estructuras ocupacionales asociadas a los cambios introducidos por la tecnología en la economía han socavado las coaliciones electorales tradicionales que sostienen el crecimiento de la administración. El votante medio, según este argumento, tiene ahora una posición nueva (superior) en la escala de distribución de los ingresos y ya no está dispuesto a pagar el precio de mantener los programas y estructuras del Estado de bienestar.

Otros, como el analista del Banco Mundial Malcolm HOLMES y el de la OCDE David SHAND (que ahora trabaja para el Fondo Monetario Internacional), dicen que la reforma de la gestión del sector público ha sido una reacción a los consabidos excesos del Estado de bienestar, tanto a nivel macro, como se refleja en el creciente tamaño de la administración y los déficit fiscales asociados, como a nivel micro, en el ya sabido reconocimiento de los límites de la capacidad del gobierno para solucionar todos nuestros problemas. ${ }^{10}$. No se tiene que estar de acuerdo con el término excesos" para aceptar el golpe certero de esta explicación. Muchas prestaciones en el moderno Estado de bienestar, concretamente las pensiones de jubilación, han llegado a ser más difíciles de pagar a lo largo de los años simplemente porque la longevidad cada vez mayor ha variado el ratio de población empleada y población jubilada. Este problema se agudizará más 
cuando se jubile la generación del baby boom. Y es indudable que actualmente la gente es más escéptica ante la capacidad del gobierno para resolver muchos problemas de la sociedad, aunque esto se debe parcialmente a que el gobierno se ha orientado hacia problemas cada vez más difíciles a lo largo de los años.

Ciertamente, como resume sucintamente la situación un reciente análisis, a comienzos de los 80 lcuando empezó a florecer el replanteamiento del gobiemo a gran escala, conocido como Nueva Gestión Públical el estancamiento, la inflación, el desempleo y la disminución de la productividad y la inversión habian afectado seriamente a los presupuestos, teniendo como consecuencia déficits cuantiosos y permanentes. ". La reducción del gasto, los elementos más pequeños del estado de la NPM eran particularmente atractivos en este entorno donde prometieron limitar el crecimiento de los gastos públicos, ayudar a reconducir los problemas del déficit sin necesidad de las nada populares subidas de impuestos y contribuir a la revitalización del sector privado ${ }^{12}$.

Otra explicación, de nuevo concretamente para la reducción de los costes y las partes más pequeñas del estado de la NPM, es que los gobiernos están perdiendo autonomía respecto de las políticas públicas debido a la globalización ${ }^{1 .}$. La competición global y el asombrosamente rápido movimiento de capital que es posible en el mundo contemporáneo hace que los gobiernos compitan más intensamente que antes respecto del capital y la inversión. Para ello, necesitan controlar los presupuestos y restringir la imposición que es la sabia del Estado de bienestar.

Los Estados hacen recortes de plantilla, según Richard RosECRANCE, para lograr ser competidores eficientes en una economía global caracterizada por el flujo cada vez más libre de tecnología, capital y trabajo. Sostiene que el flujo de los factores de producción internacionales está inundando el poder económico nacional y haciendo que el Estado confie en las fuerzas externas para resolver los problemas económicos internos:

Los países deben intentar que el capital extranjero entre en su territorio. Para mantener tal inversión, las autoridades nacionales necesitarán mantener baja la inflación, incrementar la productividad, tener una moneda fuerte y una fuerza de trabajo flexible y formada. En ocasiones, estas demandas entrarán en conflicto con los intereses nacionales que quieren un mayor gasto del gobierno, déficits presupuestarios mayores y más prestaciones. Ese conflicto dará lugar a una continua inseguridad interna sobre el empleo, la riqueza y la atención médica. A diferencia de los remedios aplicados en las economías aisladas y parcialmente cerradas del pasado, en puridad las políticas nacionales ya no pueden resolver estos problemas ${ }^{\text {. }}$.

Además de estos factores, parece obvio que el público, especialmente en los Estados Unidos, ha llegado a estar cada vez menos satisfecho con la forma en que muchos programas reguladores se han puesto en práctica. No es un asunto sencillo. La situación es, a grandes rasgos, análoga a las cuestiones del tstado de bienestar donde muchos quieren grandes beneficios y bajos impuestos. La gente quiere simultáneamente los beneficios generados por muchos regímenes reguladores -tales como aire y agua limpios y alimentos y medicamentos seguros - sin exigencias molestas. Y a menudo no les gustan los objetivos que representan las regulaciones en áreas tales como la acción positiva. El tono antirregulador del Análisis de la Actuación Nacional puede que no derive de la misma fuente que la reacción pública a la regulación, pero los líderes políticos aprecian su gran atractivo.

Finalmente, los cambios en la tecnología y en el entorno económico y los muchos problemas a los que se ha enfrentado el Estado, han contribuido aparentemente a extender el sentimiento de que los métodos empleados en el sector privado son superiores a los empleados en el sector público. Cuando los defensores de la Nueva Gestión Pública reclaman la privatización, mayor subcontratación, desregulación y cosas semejantes, están en consonancia con una corriente de opinión política que ha mostrado recientemente una gran vitalidad en gran parte del espectro políico.

En resumen, hay una variedad de explicaciones por las cuales la Nueva Gestión Pública (incluida la versión americana, el Análisis de la Actuación Nacional) ha surgido y ha llegado a ser una fuerza tan potente en el gobierno contemporáneo. Hay probablemente parte de razón en cada uno de los factores explicativos propuestos, aunque, como Hood, nos mantenemos escépticos respecto a que el argumento o las evidencias a favor de alguno de ellos es dominante o lo suficientemente irresistible como para establecer cómodamente una generalización. Ésta es una de esas áreas que demandan más investigación. Lo que está meridianamente claro es que los ejecutivos en los Estados Unidos y en la mayoría de los países industrializados se enfrentan a un entorno cambiante favorecido por los esfuerzos para implementar muchas de las propuestas expuestas por los defensores de la NPM.

Según Donald KETTL, un importante estudioso de la Nueva Gestión Pública, la revolución global de la gestión pública ha tenido dos variantes básicas. En una a la que él denomina -dejar que los gestores gestionen. y que se empleó en países tales como Australia y Suecia, "Los reformadores ... creían que los gestores sabían lo que se debía hacer, pero que las reglas, procedimientos y estructuras existentes creaban barreras para hacerlo. ${ }^{15}$. Los gestores, argumentaban los reformadores, estaban constreñidos por los procedimientos operativos estándar y limitados por su visión de lo que podrian hacer las prácticas y las políticas públicas existentes. Las reformas se deberían diseñar para liberar a los gestores de tal forma que pudiesen centrarse 
en los problemas que deberían resolverse y reaccionar ante ellos de forma flexible y creativa. •En el núcleo de deja que los gestores gestionen:, dice KETr,, "está el movimiento del servicio al cliente que centra a los gestores en servir a los ciudadanos en vez de centrarse en las necesidades de la burocracia, en la parte de 'trabajar mejor' en el dilema de 'trabajar mejor/menor coste'. ${ }^{16}$.

La segunda variante KETTL la titula baciendo que los gestores gestionen. Este enfoque se identifica concretamente con las reformas en Nueva Zelanda y Gran Bretaña. Los reformadores enfatizaron que porque la mayoría de las agencias y programas del gobierno son monopolios, los gestores tenían pocos incentivos para gestionar mejor. La única forma de mejorar la actuación del gobierno, según creían, era cambiar los incentivos de los gestores del gobierno, sometiéndoles a las fuerzas del mercado. ${ }^{18}$. Se ha usado una variedad de técnicas. En la iniciativa de los Próximos Pasos (Next Steps) de Inglaterra, se establecieron agencias (separadas de los departamentos marco de las políticas públicas) para llevar a cabo políticas públicas mediante contratos en los que se especificaban los objetivos y los estándares de gestión. En Nueva Zelanda, a los gestores se les contrata actualmente en virtud de contratos de gestión y se les puede despedir si sus unidades no logran los estándares de gestión fijados para ellos. Ambas naciones han privatizado programas y subcontratado, como elección racional que los economistas aconsejan.

KETTL sostiene que las filosofías de adejar que los gestores gestionen $\mathrm{y}$ ahacer que los gestores gestionen. van en direcciones opuestas" 19 . El enfoque de dejad que los gestores gestionen incrementa la responsabilidad de los gestores ante los consumidores. Les autoriza para actuar con flexibilidad para mejorar el servicio, todo ello al tiempo que se guían por la demanda y preferencias del consumidor. El enfoque de hacer que los gestores gestionen establece unos objetivos desde arriba y a los gestores se les otorga libertad (con algunos límites, desde luego) para hacer lo que sea necesario para conseguir los objetivos. KETTL observa que los americanos que esbozaron la iniciativa de la reinvención la tomaron prestada, en gran medida, tomando en cuenta ambos enfoques como fuente de ideas sin prestar mucha atención a cómo encajaban una con otra. El resultado es un conjunto de propuestas con muchas facetas, pero sin mucha coherencia.

¿Cuáles son exactamente los principales elementos de la iniciativa de la reinvención en los Estados Unidos? A primera vista, la iniciativa parece bastante simple. El Informe inicial del NPR lista cuatro sprincipios. clave y después organiza sus propuestas dentro de aquellos principios. Se supone que se centran principalmente en el cómo del gobierno (cómo debería trabajar el gobierno). Como senalamos anteriormente, un conjunto de propuestas posterior, denominado a menudo NPR I, otorga un mayor énfasis al ‘qué del gobierno (lo que el gobierno hace - y lo que debería hacer ${ }^{20}$. Y la última fase, NPR III, o Fase III usando la denominación de KETT, se centra en mejorar las 32 agencias de alto impacto y en usar una medición de los resultados para transformar la gestión federal. ${ }^{21}$.

Los cuatro principios clave de NPR I son los siguientes: 1) Cortar la cinta roja; 2) Poner a los clientes en primer lugar: 3) Dar poder a los empleados para obtener resultados; y 4) Volver a los elementos básicos. A pesar del énfasis en el ‘cómo", lo último tiene claramente fuertes implicaciones para el 'qué. del gobierno.

Cortar la cinta roja trae consigo una gran serie de pasos para desregular y modernizar la administración. En lo que ha recibido más atención por parte de los analistas, el informe NPR sugiere poner fin a las regulaciones a nivel federal y reducir las regulaciones federales para los Estados y entes locales. Esto incluye el proceso propuesto para permitir que las agencias puedan renunciar a las regulaciones y la sugerencia de que el Congreso imponga unos pocos mandatos a las agencias, por ejemplo, limitar lo que la crítica gusta de llamar micro-gestión congresista. También incluye propuestas para permitir que los Estados y entes locales consoliden programas de subvenciones individuales y permitir a los secretarios de gabinete conceder a los Estados y entes locales exenciones respecto de las regulaciones federales. El informe NPR también sugiere minimizar muchas restricciones en el gasto de las agencias impuesto por la OBM y el Congreso, permitiendo los deslizamientos presupuestarios entre los años fiscales, y modernizar el proceso presupuestario introduciendo la presupuestación bianual. Defiende la descentralización de la gestión del personal para dar a los gestores más autoridad para contratar, promocionar, recompensar y despedir a los trabajadores. Demanda realizar una reforma del proceso de aprovisionamiento, especialmente eliminando las rígidas reglas de aprovisionamiento y confiar más en el mercado. Y, finalmente, sugiere que la Inspección General cambie sus orientaciones sobre el cumplimiento de las normas (castigar a los transgresores) para mejorar la actuación de la agencia.

El informe NPR contiene numerosas sugerencias para colocar a los clientes en primer lugar. A las agencias se les exigirá captar el punto de vista de los clientes (empleando encuestas y técnicas similares). Esto se llevará a cabo para competir por los clientes, alli donde sea posible, con las agencias internas de prestación de servicios cuya misión es competir unas con otras y con proveedores externos de servicios, y con agencias de servicios orientadas hacia el exterior creadas para competir con las compañias privadas que pueden llevar a cabo tareas similares. Cuando un monopolio del gobierno se considera absolutamente necesario, como en el caso del sistema de control del tráfico aéreo, a las organizaciones se las debería dirigir más como empresas de negocios y se las debería dar incentivos para responder a los clientes. Finalmente, el informe insta a que se 
trasladen algunas funciones federales al mercado, incluyendo la formación continua de trabajadores y la inspección de trabajo.

El NPR contiene muchas propuestas que, como dice, conierirán poder a los empleados para conseguir resultados y crear una cultura de espíritu empresarial público. ${ }^{2}$. Debería descentralizarse el poder de decisión política, dando a los empleados de nivel inferior más autoridad para tomar decisiones de la que tienen actualmente, al tiempo que reducir el número de personal supervisor. A los empleados se les debe hacer responsables de los resultados. Aquí el NPR abraza de forma entusiasta la ley de Actuación y Resultados del Gobierno de 1993 (GPRA) y reclama su total implementación, la cual incluye planes estratégicos, planes de gestión anual con objetivos medibles y oportunidades para eximirse de las regulaciones administrativas (.renuncias para la flexibilidad de la gestión.). NPR también reclama que el Presidente desarrolle acuerdos de gestión por escrito con los responsables de los departamentos y de las agencias. El informe utiliza los contratos de gestión de Gran Bretaña como ejemplo, y eso implica que los responsables de las agencias (y las agencias) que no logren conseguir los objetivos acordados sufrirán consecuencias fatales ${ }^{23}$. Finalmente, el informe NPR pide que los empleados federales en el gobierno reinventado reciban las herramientas y la formación que necesitan para realizar su trabajo, e insiste de forma acentuada en que «se aseguren de que las utilizan. ${ }^{24}$.

El último principio de NPR I es volver a los elementos básicos. Ésta es la sección del informe que solapa el "quér del gobierno supuestamente reservado al NPR II. El primer elemento de volver a los elementos básicos especifica que el gobierno federal debería "eliminar lo que no necesitamos ${ }^{25}$. Obviamente, lo que no necesitamos está, al menos parcialmente, en función de los propios valores e intereses políticos. El informe se centra en lo que denomina lo obsoleto, la duplicación (incluido el solapamiento de programas) y la eliminación de aprivilegios de especial interés. ${ }^{26}$. Los ejemplos que el informe del NPR da de estos últimos deberían considerarse en su mayoría como no controvertidos, pero algunos pondrían una gran parte de los programas federales bajo esta rúbrica. Otras sugerencias en el apartado de volver a los elementos básicos es incrementar las tarifas a los usuarios (y dar a las agencias mayor libertad en este sentido), trabajar más concienzudamente para eliminar el fraude, invertir en una mayor productividad y redisenar la ingeniería de programas para recortar costes.

El NPR I preveía un ahorro de 108 billones de dólares durante cinco años, de los cuales casi un $40 \%$ era a través de la -modernización. de la burocracia, eliminando cerca de 252.000 puestos de trabajo (más tarde el Congreso lo incrementó a 272.9000). El NPR II preveía un ahorro de casi 70 billones de dólares en cinco años mediante cambios, finalizaciones y privatizaciones de los programas.
Un análisis que realizamos del ahorro NPR II indica que de los cambios específicamente situados (algunas agencias, como la NASA: no quebrantaron el ahorro propuesto por el progra$\mathrm{ma}), 84 \%$ precisó de cambios legislativos 27 . El ahorro, en otras palabras, no se puede alcanzar simplemente mediante cambios administrativos. Muchas de las ineficiencias en la administración vienen dadas por mandatos legislativos y sugieren una fuente política más que administrativa, de los problemas. Volveremos a esta cuestión en la conclusión de este artículo, donde enfatizamos el significado político y las implicaciones de la reinvención ${ }^{28}$.

El NPR I fue muy criticado por el énfasis que en la práctica se dio al ahorro sobre la mejora de la gestión y por su falta de estrategia a la hora de negociar con el Congreso, cuyo apoyo se necesitaría en muchos sentidos, incluida la legislación ${ }^{29}$. El Presidente y el Vicepresidente anunciaron reducciones en las dimensiones de la burocracia y el ahorro proyectado en los costes. En resumen, la parte del costar menos- del NPR dominó sobre la parte de «trabajar mejor , sin que sorprendiese a ningún observador político, pero generó un problema desde entonces, como muy bien puso de manifiesto Donald KeTTL: buscar un gran ahorro a corto plazo puede socavar un esfuerzo mayor de mejorar la gestión e incrementar los costes a largo plazo. ${ }^{30}$.

En suma, el NPR es parte de un amplio movimiento conocido como la Nueva Gestión Pública (New Public Management). Mientras que tiene muchas variantes, todas comparten la idea de que se debe cambiar la administración y la cultura administrativa centrándose en los resultados organizacionales, la gestión individual y los incentivos del mercado. Algunos enfatizan el enfoque orientado al cliente que KeTtr titula como dejar que los gestores gestionen, mientras que otros se centran en el enfoque más controlado centralmente de hacer que los gestores gestionen", donde las autoridades centrales establecen objetivos y a los gestores se les da una gran libertad para diseñar las formas de lograrlos. El Análisis de la Actuación Nacional (National Performance Review) toma prestados indiscriminadamente ambos enfoques. Pone un mayor énfasis en el servicio al cliente, pero también se caracteriza por objetivos de gestión determinados centralmente. En conjunto, el NPR se propone recortar programas, ahorrar dinero, hacer uso de la subcontratación, la privatización, la competencia y los mercados, y adoptar las técnicas del sector privado en un sistema administrativo orientado al rediseño de los resultados/gestión. También apoya la gestión de abajo arriba y se centra en la satisfacción del cliente. Dice poco sobre lo que debería ocurrir si estos elementos entrasen en desacuerdo.

Se podrían escribir volúmenes sobre las herramientas de gestión recomendadas por el Análisis de la Actuación Nacional (National Performance Review). De hecho, la gente lo hace. Y muchas de las propuestas del NPR contienen ideas interesantes 
para liberar a los gestores federales y permitirles que gestionen eficientemente. Cuando criticamos algunas de estas ideas del análisis de la gestión, como haremos en la siguiente sección, el espíritu es constructivo. De hecho, no queremos echar un jarro de agua fría en muchas de las reformas de gestión que el NPR sugiere (o al menos en la necesidad de las reformas de gestión), pero nos centraremos en las dificultades de la reforma y después enfatizaremos que el problema es más profundo de lo que el NPR o el NPM sugiere.

\section{Crítica de la reinvención: problemas y perspectivas}

Como ya se ha indicado, muchos de los elementos del NPR son atractivos. Es especialmente útil revisar el conjunto de regulaciones que a menudo frustran a los trabajadores federales y a los ejecutivos más de lo que frustran a aquellos de nosotros que tenemos un contacto directo relativamente infrecuente con los programas. Muy pocos negarían las ventajas de una reflexión cuidadosa sobre lo que podría hacerse mejor o de forma más flexible en el sector privado (aunque es probable que merezcan más la pena en otras naciones donde el sector público históricamente ha gestionado los sistemas de transporte y ha prestado otros servicios cuasi-comerciales). Una mayor subcontratación podría tener éxito (aunque gran parte de la experiencia del gobierno en esta área demuestra lo difícil que puede ser gestionar el proceso de contratación de forma eficiente y eficaz) ${ }^{31}$. Ciertamente, la mayoría de los observadores objetivos pueden aplaudir únicamente los esfuerzos que se han realizado para emplear la moderna tecnología de la información en hacer que las organizaciones sean mejor dirigidas y extenderse de tal forma que cada vez menos trabajadores den un producto mejor. $Y$ a todo aquel que le importe cómo funciona el gobierno, debería querer ver a aquellos a los que sirve recibir un tratamiento cortés, educado y satisfactorio en las oficinas del gobierno.

Nosotros no nos proponemos en esta sección criticar cada uno de los muchos cambios sugeridos por el NPR. Más bien, queremos analizar los cambios propuestos buscando las áreas que necesitan ser reelaboradas y demostrar más adelante que lo que parece atractivo a simple vista, en su interior esconde un problema. Comenzamos con una crítica de los cuatro principios básicos del NPR y después pasamos a discutir otros temas planteados por la puesta en práctica del NPR, incluyendo cuestiones más amplias que afectan al sistema político.

El principal objetivo (o principio, como a los del informe NPR les gusta denominarlo) de la reinvención es cortar la cinta rojar. Este objetivo está tan cercano a la maternidad como no se puede encontrar en el mundo frecuentemente seco de la reforma administrativa. La cinta roja es un término peyorativo en nuestro lenguaje. Hay muy poca gente proclive a ratificar que la cinta roja es algo que el gobierno necesita.

Sin embargo, un análisis concienzudo y desapasionado de la cinta roja realizado por Herbert Kaufman nos haría pensar dos veces en los usos de la cinta roja, así como en sus abusos. Kaufman sostiene que la cinta roja (que rodea los constreñimientos oficiales en cuanto al comportamiento) se creó para proteger a la gente de los abusos, para garantizar un procedimiento ajustado a las normas (justicia), para asegurar que los intereses pueden estar representados en el proceso político (y de una forma ordenada y racional), para promover la eficacia en la administración y para asegurarse de que las acciones adninistrativas están abiertas al escrutinio público ${ }^{32}$. Éstos son valores que la mayoría de la gente en una sociedad democrática aprueba. Desafortunadamente, sin embargo, los procedimientos para promoverlos a veces hacen que las cosas sean molestas o irritantes. Es el dilema de la cinta roja -sopesar los costes y los beneficios. El NPR, para simplificar la cuestión, está abierto a la crítica de que se debe considerar los cambios procedimentales propuestos con mucho cuidado, incluidos los propuestos con el loable esfuerzo de desregular y modernizar la administración, para asegurarse de que los costes, así como los beneficios esperados se tienen en cuenta.

James Q. WIISON, siguiendo a KAUFMAN, sostiene que las garantías de justicia y previsibilidad en el sector público, como las nomas que regulan cómo alquilan, compran y contratan las agencias, otorgan una protección fundamental a los ciudadanos y a los grupos de interés en nuestra sociedad. Las normas garantizan que existen procedimientos establecidos para desarrollar y promulgar regulaciones (la Ley de Procedimiento Administrativo), de tal forma que la gente que tiene interés puede saber lo que el gobiemo planea hacer y tener algo que decir. A veces, las reglas se establecen para asegurarse de que un grupo concreto tiene acceso a los recursos públicos (negocios pertenecientes a minorías o gobiernos locales son los dos ejemplos que WILSON utiliza). Aquí las normas se emplean para proteger un interés o dejar constancia del vencedor de una disputa política ${ }^{33}$.

La conclusión obvia es que es probable que la flexibilidad administrativa sea considerada como una virtud hasta que dañe algún interés básico. Entonces se demandará poner de nuevo en práctica procedimientos que aseguren la justicia, la transparencia, la previsibilidad y los valores relativos. Como Peter Aucorn señala en un ensayo sobre la reforma administrativa en la gestión pública que se centra en otros paises además de Estados Unidos: ‘Los tests cruciales son si los políticos, especialmente las autoridades ejecutivas, se autolimitarán a la hora de pedir nuevas regulaciones o de volver a regular cuando tengan lugar los inevitables errores administrativos y, por tanto, la tur- 
bación política o cuando se descubran las desviaciones de las normas recientemente aceptadas, tales como la equidad en el empleo $^{3:}$. Precisamente por este enfoupe eñ los sistemas parlamentarios de tipo Westminster, el ensayo de Aucoln no considera la posibilidad todavía mayor de que los políticos elegidos de una institución como el Congreso de los Estados Unidos, demanden rápidamente reglas y procedimientos en respuesta a los escándalos y abusos. De hecho, muchas de las reglas más restrictivas que tenemos hoy dia están en vigor debido a acciones del Congreso.

El segundo principio del NPR es aponer a los clientes en primer lugar. Éste es otro objetivo que parece simple y deseable a primera vista. Naturalmente, queremos que las agencias del gobierno satisfagan a aquellos que sirven y les den el mejor tratamiento posible. Sin embargo, no todos los clientes son iguales.

Cuando una agencia del gobierno, como el Servicio Postal, tiene como clientes a toda la ciudadanía (y los clientes están pagando unos precios por los servicios recibidos), no es probable que haya mucho debate acerca de la prestación de los servicios según las preferencias y demandas de los clientes. Pero a menudo, los clientes tienen regulados sus intereses o son receptores de beneficios (tales como los del Estado de bienestar). Muchos otros dentro de la sociedad están en juego en estas áreas políticas. Pueden ser los proveedores de fondos mediante la redistribución de ingresos fiscales generales o estar afectados por los intereses regulados (tales como las emisoras o los procesadores de alimentos). ¿Cuáles son los clientes apropiados a tener en cuenta en estos casos? ¿A qué intereses se les debería dar primacía? Éstas son básicamente preguntas sobre la legitimidad e igualdad y son problemas políticos de primer orden. En resumen, no siempre está claro quiénes son los clientes y a quién se le debería "colocar en primer lugar".

Una crítica afín al principio de poner a los clientes primero es la forma en la que se concibe (o más exactamente, no se concibe) a los ciudadanos. Algunos analistas incluso llegan a caracterizar la reinvención como una negación de la ciudadanía. El argumento, expuesto brevemente, es el siguiente: -Como ciudadanos, esperamos del gobierno que actúe de una forma que no sólo promueva el consumo de servicios ... sino que también promueva un conjunto de principios e ideales que son inherentes a la esfera pública ${ }^{35}$. Hay una diferencia, en otras palabras, entre los proveedores de productos del sector privado y esas cosas que nosotros nos esforzamos por hacer colectivamente a través del gobierno.

Una figura importante en el diseño de la iniciativa de la reinvención, John KAMENSKY, trata indirectamente esta crítica, al realizar una distinción entre permitir que los clientes expresen su opinión sobre la prestación de un servicio y darles la palabra en la elaboración de las políticas públicas. Sostiene que el NPR defiende incrementar el rol y la voz de los clientes en la presta- ción del servicio (pero no en el proceso de elaboración de las políticas públicas. ".t. Los líderes del gobiemo deben establecer un marco político básico dentro del cual se pueda perseguir la satisfacción del cliente. Esta distinción deberá ayudar en muchos casos, pero deja a un lado la contradicción básica discutida anteriormente en este articulo - la tensión entre lo que los clientes pueden desear y lo que la política del gobierno requiere-, particularmente si a los gestores se les evalúa en base a criterios de gestión más allá de la satisfacción del cliente.

Finalmente, hay alguna duda sobre si las analogías del mercado propuestas por el NPR sobre el servicio al cliente son enteramente exactas. La presuposición del NPR es que las organizaciones del sector privado tienen como principal objetivo conseguir beneficios. Satisfacer a los clientes es un medio para conseguir un fin, pero no es un fin en sí mismo. Cuando las empresas pueden hacerlo, eliminan la competencia y exprimen a los clientes lo que pueden. E incluso cuando hay competencia, las empresas deben calibrar la compensación entre lo que a los clientes les gustaría (servicios excelentes) y lo que prefieren pagar (precios bajos). Cualquiera que vuela con líneas nacionales conoce muy bien este intercambio.

En resumen, existen muchos problemas todavía sin resolver respecto de la atractiva idea de poner a los clientes en primer lugar.

- Dotar de poder (empowering) a los empleados para conseguir resultados n es el tercer principio del NPR. Descentralizar la autoridad hasta maximizar el potencial de los trabajadores del gobierno para contribuir de forma creativa a mejorar la gestión organizativa es otro objetivo que pocos discutirian. Como los otros objetivos del NPR, no obstante, surgirán dificultades significativas a la hora de lograr adar ese poder a los empleados. La primera y más obvia es el riesgo a los escándalos. WILSON cita la siguiente frase del informe NPR, con la que está de acuerdo: -En el altamente politizado mundo de Washington, el mayor riesgo no es que un programa se desarrolle de una forma pobre, sino que estalle un escándalo ${ }^{37}$. Él presupone, razonablemente a nuestro entender, que esta dinámica continuará funcionando. No es un campo fértil para que tenga éxito conferir poder (empowerment) a los empleados.

Otra dificultad con el empowerment de los empleados es el problema de lograr un acuerdo sobre las expectativas. De nuevo volvemos a la tensión entre los objetivos definidos por los empleados (y los clientes) y los objetivos de los funcionarios elegidos o los que toman las decisiones al más alto nivel en la jerarquía administrativa organizacional. ¿Cuánta flexibilidad deberían dar los sistemas democráticos a los empleados de la administración, especialmente desde que la administración política define con frecuencia lo que es la política? Si el sistema político da a los administradores objetivos vagos, como a menudo ocurre, entonces, ¿cuánta libertad tendrían los empleados del go- 
bierno respecto de la interferencia (supervisión) que puedan ejercer las autoridades centrales? ¿Y qué hacemos cuando el Congreso y el ejecutivo no están de acuerdo en los objetivos y después dan señales opuestas sobre cuáles deberían ser los objetivos o el comportamiento de la agencia?

Muchas de estas preguntas se plantearán de nuevo cuando discutamos la implementación inicial de la ley de Actuación y Resultados del Gobierno (Government Perfornance and Results Act -GPRA). El informe NPR menciona la GPRA como un excelente ejemplo de empowering a los empleados para lograr los resultados y hacerles después responsables ${ }^{38}$. Como en la mayor parte de la discusión anterior, deberíamos hacer constar desde el principio que hay grandes ventajas en desarrollar objetivos medibles para que los empleados de la agencia los alcancen. Los problemas vienen al implementar una ley como la GPRA en situaciones que se dan comúnmente, donde los objetivos no se han establecido bien políticamente o donde existe más o menos un acuerdo sobre los objetivos, pero el nivel de recursos de los programas puede estar en discusión (a menudo, desde luego, tanto los objetivos como los recursos están en disputa).

El cuarto y último objetivo del NPR es "volver a los elementos básicos" (eliminando "lo que no necesitamos" como primer paso). Ante el riesgo de parecer un disco rayado, ¿cuántos podrian discrepar en principio de la idea de que deberiamos elininar lo que no necesitamos? Sin embargo, podría existir cierta disputa sobre lo que debería incluirse en lo que es básico. De hecho, sostendremos que el núcleo del problema, mucho más importante que las cuestiones de los sistemas o mecanismos administrativos, es alcanzar el consenso sobre lo que el gobierno debería estar haciendo.

Como James Q. WILSON senala, antes que cerrar algunas oficinas de la agencia y hacer sugerencias similares que no es probable que provoquen muchas iras, el Informe inicial NPR establece como objetivo subvenciones a la lana, a las abejas y al mohair ${ }^{39}$. Éstas son sugerencias relativamente seguras, aunque probablemente contestadas por los partidarios de los programas o quienes consideran útiles o convenientes las oficinas. Como señalamos anteriormente, el NPR II hace sugerencias más sustanciales a los programas, pero la mayoría de sus propuestas requieren de la actuación del Congreso, porque la legislación ha establecido los programas o formas de prestación de los servicios que son objeto de supresión o cambio. La clave para hacer los cambios es, pues, política y no administrativa. Hay distritos de apoyo que están tras la mayoria de los programas del gobierno. Los seguidores insisten regularmente en que la nación necesita sus programas favoritos ( $\mathrm{y}$ a menudo tienen sugerencias para mejorar los programas, extendiéndolos de alguna forma). A menudo, trae consigo el conflicto y gasto de capital político decidir lo que es básico y lo que no necesitamos, aun- que, con frecuencia, pueden ayudar también circunstancias afortunadas como las consecuencias de la caída de la Unión Soviética para el programa de defensa de Estados Unidos.

Otros analistas hacen alegaciones similares. Ron MOE, por ejemplo, en un duro análisis del NPR, concluye que después de todo lo que se ha dicho y hecho to que realmente necesitamos hoy día ... es volver a pensar lo que realmente esperamos de nuestro gobierno " ${ }^{40}$. George FrEDERICKSON sostiene que reinventar el gobierno está plagado del sproblema de los problemaserróneos. Dice que een el problema de los problemas-erróneos, un gobiemo busca evitar tomar decisiones políticas duras que tengan que ver con los problemas públicos reales y, en vez de eso, se vuelve hacia una mejor gestión como la solución universal. ${ }^{41}$. Desde luego, el NPR sugiere algunas elecciones políticas, pero muy pocas son realmente duras. Y son estas duras elecciones a las que FREDERICKSON se refiere cuando habla de los aproblemas públicos realesn.

Precisamente porque el NPR contiene tantos elementos y toca tantos aspectos importantes del gobierno, se podría continuar casi ad infinitum haciendo comentarios y críticas. Haremos algunas aclaraciones adicionales (y explicaremos además unas cuantas que ya se han planteado) en las siguientes páginas, pero de nuevo ponemos de manifiesto que, al hacer eso, no negamos muchas de las útiles sugerencias del NPR para hacer operativo el gobierno de una forma más sensible y eficiente.

Para un paquete de reformas que tiene que ver con la eficiencia y la forma en la que las organizaciones del gobierno funcionan, el NPR presta relativamente poca atención al rol de los directivos de nivel superior. El NPR demanda recortes entre el personal supervisor de nivel medio como medio de reducir el tamaño del funcionariado público e incrementar simultáneamente el grado de control, pero curiosamente guarda silencio sobre aumentar el tamaño del cuerpo de los nombrados políticamente. Sus cifras se han incrementado dramáticamente desde 1960, parte de un fenómeno al que Paul Licht se refiere como el "engordamiento del gobierno* '2. Un análisis del libro de LiGHT describe bien el problema:

Como [LIGHT] señala tan acertadamente, las anteriores justificaciones de la limitada envergadura del control descansaban en las limitaciones innatas de los líderes; las motivaciones actuales para ese engordamiento descansa en el deseo de los presidentes de ejercer un control más estrecho sobre los burócratas en los cuales no confían. Pero sin dirigirse de forma sistemática en sus recomendaciones a los nombrados políticamente, el informe Gore no llega a eliminar un importante obstáculo para conseguir una burocracia federal más reducida y que lleve a cabo una mejor gestión ${ }^{43}$. 
Un problema es que con tantos cargos políticos en el más alto nivel, es difícil hacer responsable de los resultados a una persona en concreto —uno de los objetivos de! NPR-. Otro, puesto de manifiesto por la Comisión Volcker, es que los conocimientos y la experiencia de los oficiales de carrera superiores no son utilizados adecuadamente cuando los cargos políticos mantienen tantas posiciones clave ${ }^{44}$. El NPR dice mucho de la contratación, formación del personal y uso de la moderna tecnología, pero se aleja del problema políticamente sensible de que haya probablemente demasiados cargos políticos presidenciales, muchos de los cuales carecen de las aptitudes de los funcionarios de carrera que están por debajo de ellos.

KETTL resalta otra pieza perdida en el NPR, la necesidad de enlazar el sistema de gestión reinventado con el presupuesto. Una enorme ventaja potencial de un sistema de gestión basado en la actuación es que puede ayudar a clarificar cuando se piensa en los objetivos políticos y suministra datos sobre si se están consiguiendo esos objetivos. Eso, a su vez, ayudaría a los funcionarios electos a sopesar las demandas en liza respecto de los recursos escasos y a poner el dinero donde sea mejor. " ${ }^{\text {is }}$. La Ley de Actuación y Resultados del Gobierno (Government Performance and Results $A c t)$ se dirige ciertamente a tener un efecto profundo en el lado de la ecuación de la clarificación y medición de la gestión- aunque todavía no está claro cómo funcionará finalmente en la práctica (véase la siguiente sección) y la parte de la ecuación del presupuesto todavía es bastante problemática. De hecho, la Auditoría General informa, de una forma comedida, de que .debido a la gran variación entre las estructuras de actividad de los programas de las agencias es probable que se cuestionen al Congreso y al ejecutivo cuando traten de vincular los objetivos de la gestión con las estructuras de actividad de los programas del presupuesto ${ }^{16}$.

Podríamos seguir en este sentido, pero no hay duda de que existen numerosos problemas con el anteproyecto del NPR (y con el NPM), que requieren una cuidadosa meditación y difíciles elecciones. Cuanto más consistentes son los elementos de la reforma, mayores serán las posibilidades de que tenga éxito. Algunas propuestas pueden llevarse a la práctica fácilmente y muchas lo han sido. Parecen haberse implementado con cierto éxito todas las reformas, mayor subcontratación, modernización del proceso de contratación, el uso de diversos mecanismos para calibrar la opinión del cliente de la agencia y responder a ello, un uso mayor y más efectivo de la tecnología de la información, la modernización de algunos aspectos del aprovisionamiento y atención a una variedad de reformas de gestión interna de las agencias ${ }^{\text {i7 }}$. La cuestión a largo plazo es si se plantean y resuelven las inconsistencias y problemas con el NPR identificadas anteriormente.
De especial atención son las siguientes cuestiones:

1. ¿Cómo se conciliarán los elementos de dejar que los gestores gestionen $y$ thacer que los gestores gestionen?

2. ¿Cómo responderá el sistema político de los Estados Unidos, sensible a los errores, a las inevitables equivocaciones y escándalos más importantes que tendrán lugar en un gobiemo reinventado tal y como tienen lugar en el sector privado?

3. iPuede ser también responsable una burocracia tan flexible y adaptable como la que visionaba el informe NPR y ante quién? KETTL, que ciertamente no se opone a las reformas, va tan lejos como para decir que la medición de la gestión y el énfasis en los clientes de la reinvención/propuestas del NPM transforma sutilmente la relación entre los funcionarios elegidos y los administradores. $\mathrm{Al}$ menos, introduce nuevos estándares por los cuales los administradores pueden afirmar su autonomía de los que elaboran las políticas públicas; a lo sumo, desajusta las influencias existentes que los funcionarios electos usan generalmente para afirmar su control sobre los gestores públicos ${ }^{\text {}}$.

4. ¿Cómo fodría tratar el gobierno reinventado con las inconsistencias inherentes a la separación americana del sistema de poderes, especialmente cuando existe un control distinto por parte del Congreso y la presidencia? ¿Ante qué institución deberían ser responsables las agencias por los resultados? ¿Qué pasaría si el Congreso no consiguiese un acuerdo apropiado sobre la financiación necesaria para lograr los resultados especificados en los planes de la agencia? ¿Entonces, cómo se evaluaría a los gestores?

5. ¿Cómo sería la relación entre los gestores y los funcionarios electos en un NPR implementado? ¿Estarían dispuestos los oficiales electos, especialmente el Congreso, a abstenerse de interferir en la administración, incluso asumiendo que existe un acuerdo sobre los objetivos? De una forma más amplia, ipor qué estaría dispuesto el Congreso a aceptar en la práctica el limitado rol que implica la iniciativa de la reinvención? ${ }^{49}$.

6. ¿Cuáles serán, si es que las hay, las consecuencias políticas y sociales si, como parece ser el caso, el énfasis a corto plazo en la eficiencia de reinventar el gobierno ha impuesto un peaje en la equidad social.? ${ }^{50}$. ¿Se acercará el énfasis en lo básico y la magra filosofía estatal y los mecanismos de reinventar el gobierno a las demandas cada vez mayores de equidad que es probable que acompañen a la siguiente recesión económica importante? 


\section{La GPRA: dilemas de la reinvención}

El Congreso aprobó la Ley de Gestión y Resultados del Gobierno (Government Performance and Results $A c t$ ) y el Presidente Clinton la firmó en 1993, el año del informe inicial del National Performance Review. El informe NPR adopta esta ley. Lo ve como una herramienta esencial para conseguir que las agencias establezcan objetivos medibles para sus programas y para hacer responsables a los máximos gestores de sus resultados ${ }^{\text {'l }}$. Y como señala KeTTL, la GPRA es una piedra angular del NPR III dado el énfasis que pone en lograr los resultados en 32 "agencias de alto impacto" ${ }^{52}$. Es demasiado pronto para pronunciar una opinión sobre la ley, pero los primeros resultados informan de los problemas habidos en la implementación de un elemento clave del National Performance Review/New Public Management en el contexto de los Estados Unidos.

La GPRA tiene por objeto incentivar una mayor eficiencia, efectividad y responsabilidad en el gasto federal, estableciendo un nuevo marco para la gestión y, en consecuencia, en la presupuestación de las agencias federales. El marco exige que las agencias establezcan objetivos, midan la gestión e informen de los resultados" ".3. La Ley exige que las agencias creen planes estratégicos (que cubran un período de cinco años: actualizados al menos, cada tres años), planes anuales de gestión e informes anuales sobre la gestión de los programas ${ }^{54}$. Los planes de gestión deben incluir objetivos de gestión expresados ade una forma objetiva, cuantificable y medible y uestablecer indicadores de gestión que se empleen para medir o valorar los outputs relevantes, los niveles de servicio y los resultados de cada actividad de los programas. "i;. Las agencias deben consultar al Congreso y al OMB y solicitar los puntos de vista de otros en el desarrollo de sus planes estratégicos. La Ley también permite al Director del OMB eximir a los programas de ciertas reglas y normas no estatutarias, si las exenciones ayudan a mejorar los resultados de los programas ${ }^{36}$. Se designaron cinco agencias como pilotos para probar la presupuestación de la gestión para los años fiscales 1998 y 1999, facilitando asi una vinculación directa con el proceso presupuestario 57 .

Es fácil comprender el entusiasmo del informe NPR por esta Ley. Y, puesto que la Lcy reclamaba que el Departamento General Contable realizase evaluaciones ya se dispone de indicios significativos sobre su implementación, aunque la total implementación llevará siete años.

La GAO elaboró dos informes en 1998 que son particularmente iluminadores ${ }^{38}$. Los informes examinan el marco estatutario para la gestión y la responsabilidad basada en la actuación y analiza los planes estratégicos de las 24 agencias más importantes, que se enviaron formalmente al Congreso y al OMB en septiembre de 1977 en el primer ciclo completo de la planificación estratégica. Mientras que los planes de septiembre de las agencias se valoraban mejor que las anteriores entregas, la GAO hizo numerosas críticas. De hecho, los planes estratégicos se describieron con extrema delicadeza como eun trabajo todavía muy en progreso. 59 .

Los problemas con los planes son bastante reveladores. Algunas dificultades son simplemente decepcionantes, pero no demasiado sorprendentes para las primeras etapas de tal esfuerzo. Muchas agencias, por ejemplo, listaron los objetivos que no se centraban en resultados factibles" y los objetivos no "siempre se expresaban de una forma que condujese a valorar el progreso en términos de la actuación real. Con frecuencia, las agencias tampoco discutían cómo iban a cumplir los objetivos ${ }^{60}$. Posiblemente, el tiempo y la experiencia podrían resolver estos problemas, al menos en tanto en cuanto no estén relacionados con dificultades más importantes.

Sin embargo, existen indicios de que algunas deficiencias van más allá del mero ajuste al entorno cambiante. Por ejemplo, la GAO identifica dificultades de las agencias en dirigir esfuerzos en programas fragmentados y que se solapan entre sí, y en prioridades políticas contendientes en sus planes. Éstas no forman parte de los problemas administrativos de las agencias, pero se relacionan con los mandatos legislativos dados en el complejo entorno político de los Estados Unidos. Un informe de la GAO, por ejemplo, comenta el hecho de que el Servicio Forestal ha sido incapaz de reconciliar los conflictos sobre el uso de las tierras y citaba el ejemplo de promover la venta de bosques versus proteger la flora. Añade que "calibrar el correcto equilibrio es un reto continuo y difícil, porque avanzar en el consenso político necesario para crear y sostener un programa, con frecuencia da lugar a un programa que tiene objetivos opuestos y/o definidos ampliamente. ${ }^{61}$.

De hecho, lograr el equilibrio adecuado es un reto político difícil para muchas agencias federales. La GPRA deja que las agencias propongan objetivos ${ }^{62}$. Eso puede ponerlas en peligro en los comités del Congreso y los distritos, lugar éste en el que no les gusta estar a los ejecutivos inteligentes de las agencias. Apenas es sorprendente que las agencias a menudo se hayan alejado de caminar por este potencial campo de minas.

Tampoco es sorprendente que algunos miembros del Congreso hayan sido bastante críticos con los planes estratégicos de las agencias. Por ejemplo, el líder de la Mayoría de la Cámara, Dick Armey, castigó a las agencias por los fracasos en dirigir el solapamiento entre los programas federales ${ }^{63}$. Una crítica mucho menos amistosa de la posición de Armey, que escribía en el diario Government Executive, reaccionó preguntando: •Pero, por cierto, ¿quién creó todos esos programas redundantes?. ${ }^{64}$. 
Ella sugirió que el Congreso liderase el reconte de los programas redundantes esin importar a qué distrito o que grupo de presión se quería agradar creándolo.s. ${ }^{65}$. Aunquue esto no es probable, pone de manifiesto una dificultad fundamental. La mayoría de los problemas del solapamiento de los programas y responsabilidades y de los objetivos poco claros o contradictorios se remonta a la legislación que los autoriza, sobre la que se ha llegado a un acuerdo en el proceso político. La GPRA aconseja a las agencias que fijen sus objetivos de forma clara, aunque esto a veces pueda ser imposible legal y/o políticamente.

Un problema relacionado con esto es que la GPRA exige que las agencias consulten con el Congreso el desarrollo de sus planes estratégicos, pero no se dejó claro exactamente lo que se entiende por consulta ${ }^{66}$. Las agencias claramente se deben comunicar con los órganos relevantes del Congreso y tratar de alcanzar un acuerdo con ellos, pero nadie sabe con exactitud lo que ocurrirá si el Congreso o el OMB discrepan, o lo que ocurrirá en la situación bastante posible en la que las dos Cámaras no están de acuerdo. La Ley también permite que el Congreso añada, enmiende o abola un objetivo de gestión. Ésta sería la última forma de hacer que los gestores gestionen. KAISER y MCMuRTRY, del Servicio de Investigación del Congreso, señalan sin embargo que no es del todo seguro ... cómo se podría lograr cualquier sustitución de un objetivo" ${ }^{67}$. Hacer responsables a los gestores de la forma prevista por el NPR y la GPRA puede ser de hecho difícil.

Existen otros problemas significativos que es probable que surjan, incluso si se logra un acuerdo sobre los objetivos o el Congreso no pone ninguna objeción inmediata a los objetivos especificados en los informes de las agencias. Por ejemplo, Fred KAISER y Virginia MCMURTRY plantean la siguiente pregunta: :Cómo se valorará el fracaso en la no consecución de ciertos objetivos si, tras la aprobación de un plan, se reduce el presupuesto de una agencia; si la financiación se reservase para otros propósitos y, por tanto, se limitase indirectamente a ciertos objetivos; o si la reprogramación y las rescisiones afectasen a la capacidad de la agencia para lograr los objetivos todavía no conseguidos? ${ }^{68}$. Éstas son buenas preguntas y son semejantes a las planteadas anteriormente sobre los posibles problemas que podría plantear el enfoque del New Public Management cuando se aplicó en los Estados Unidos, con su complejo sistema de gobierno (de poderes separados).

Otros problemas mencionados por la GAO incluyen el hecho de que el gobierno federal a menudo tiene una influencia bastante limitada o indirecta para determinar si un objetivo deseado se ha logrado. Señala que uel trabajo de la $\mathrm{GAO}$ ha demostrado que medir la contribución federal está desafiando concretamente los programas reguladores; los programas de investigación científica; y los programas para prestar servicios a los contribuyentes a través de terceras partes, tales como los gobiernos estatales y locales ${ }^{(6)}$. Éstos son los elementos más importantes de la serie de programas del gobierno de los Estados Unidos. A la GAO también le preocupa que los datos de actuación de las agencias a menudo son de baja calidad, debido a una variedad de factores, incluidos algunos que son dificiles de controlar, tales como la necesidad de confiar en que terceras partes le suministren los datos. ${ }^{70}$. Los gestores en el contexto de la GPRA/NPR van claramente a tener que ser más hábiles que nunca a la hora de asegurar y evaluar los datos de los Estados y los poderes locales y de los muchos contratistas (adicionales) previstos en el informe NPR.

Finalmente, como señalamos anteriormente en el documento, la GAO está preocupada por las dificultades que probablemente surgirán en vvincular los planes de actuación de las agencias directamente con el presupuesto. Esto es debido al hecho de que el punto hasta el que la estructura de actividades de un programa del presupuesto puede vincularse al marco de la gestión orientada a resultados varía enormemente de unas actividades a otras. El problema no es que los ajustes son difíciles de concebir o implementar, sino que el Congreso y el ejecutivo todavía tienen que ponerse de acuerdo sobre los cambios que se necesita realizar y lograr un acuerdo, como mínimo, será un proceso difícil, que necesita tiempo y que llevará más de un ciclo presupuestario resolver. ${ }^{71}$.

A pesar de la existencia de todos estos problemas (y quizá de otros no previstos), es probable que la GPRA tenga muchos efectos positivos. A través del ejercicio de elaborar planes estratégicos, planes anuales de gestión e informes anuales de gestión, las agencias se verán obligadas a tomar en cuenta sus objetivos de una forma más clara y medible. Mejorarán el uso que hacen de la tecnología de la información y serán casi con toda seguridad mejores supervisores de los Estados, poderes locales y contratistas. La GPRA también pondrá de relieve las diferencias entre el Congreso y el ejecutivo sobre las políticas públicas y quizá estimule una mayor claridad en la legislación e impulse los esfuerzos para disminuir el solapamiento y la fragmentación de los programas. Pero, al final, los problemas más sensibles de la definición de objetivos, la fragmentación de los programas y los niveles inciertos y cambiantes del apoyo presupuestario a los programas son problemas políticos en esencia. Las reformas de la gestión pueden poner de manifiesto tales problemas, pero no pueden solventarlos.

\section{Algunas cuestiones relevantes}

Escondida tras gran parte de esta discusión del NPR yace una cuestión difícil de contestar: ¿cuál es el grado de importancia de las cuestiones de la gestión en el panorama global? La 
iniciativa de la reinvención y la Nueva Gestión Pública (New Public Managament) de la cual es parte, supone que la reforma de la gestión es una respuesta primordial a los problemas que afrontan los gobiernos actuales. El gobierno reinventado no sólo trabajará más eficientemente, dicen los reinventores, proporcionando un gobierno que trabaje mejor y con menores costes, sino que reducirá los servicios del Estado a los elementos básicos, eliminará lo que no es necesario y restaurará la confianza de la gente en el gobierno. Además, el gobierno reinventado estará más en sintonía con las ventajas de la moderna tecnología de la información que puede traer y situar al país en una mejor situación para competir en la economía globalizada.

Muchas de las propuestas del NPR son relativamente no controvertidas. Hay poco debate sobre la deseabilidad de prestar un correcto y eficiente servicio de aquellos que entran en las oficinas del gobierno o que usar la tecnología de las comunicaciones y el procesamiento de la información contemporánea hace que las organizaciones funcionen de una forma más suave y eficiente o incluso, al menos, considerar los costes y beneficios de la contratación externa y la privatización en muchas áreas. Y la mayor parte de los observadores estarian de acuerdo en que se deberian revisar cuidadosamente las reglas y regulaciones y se deberian eliminar selectivamente, aunque podría haber gran desacuerdo en qué reglas son necesarias.

Las dificultades aparecen cuando entramos en la parte politica de la reinvención. Aunque establecido como un conjunto de reformas de la gestión, el núcleo de la reinvención es político. Plantea todo un conjunto de cuestiones que a menudo encubre cambios propuestos en la técnica de la gestión o la política de personal ${ }^{72}$. El NPR propone cambios en la distribución de poder, aunque no resuelve quien ganará. Hay una tensión en el NPR entre incrementar la influencia de aquellos que están situados en el centro e incrementar la influencia de la gente que el estudio de la reinvención denomina ‘clientes. Y es comprensible que el NPR sea vago sobre quién de los que están en el centro ganará poder, aunque el informe del National Performance Review quita importancia al Congreso y, de ese modo, da una respuesta. Sobre la cuestión de la discreción y el juicio, el NPR aprueba un incremento del poder discrecional de los funcionarios de nivel inferior, pero, al mismo tiempo, se supone que dominan los objetivos de aquellos situados en el nivel superior (o las preferencias de los clientes, dependiendo de la sección del informe). Y hace presuposiciones sobre cortar la cinta roja que, como hemos visto, son altamente cuestionables en el mejor de los casos. Acerca de la responsabilidad (iquién es legalmente responsable de qué?), el NPR no es claro. Y ciertamente cuando llega a la engorrosa cuestión de la responsabilidad en el gobierno de los Estados Unidos (ia qué jefes en un sistema de poderes separados, y a menudo divididos, tienen que responder los burócratas?), la cuestión permanece sin resol- ver y sigue siendo discutible con el NPR, al igual que lo era antes. Esto no es un problema del NPR más importante de lo que lo fue en el pasado, excepto por el hecho de que se supone que el NPR incrementa la eficiencia.

Quizás el mayor problema aparece cuando tratamos de volver a los elementos básicos y eliminar lo que no es necesario. Mientras el NPR trata éstos como problemas de gestión, de hecho, los enfatiza en un contexto de gestión, tan sólo son cuestiones de poca importancia en la gestión. Van al núcleo de lo que una sociedad espera de su gobierno y lo que está dispuesta a pagar. Incluso haciendo presuposiciones optimistas sobre el incremento de la eficiencia del gobierno, las cuestiones sobre lo que el gobierno tiene que hacer y quién debe pagar siguen siendo centrales.

Los Estados Unidos, como la mayoría de las democracias de estilo occidental, han hecho frente a un conjunto de problemas políticos que se han manifestado de diversas formas desde principios de los años 70. Éstos incluyen problemas tales como la escasez relativa de recursos (mayores derechos adquiridos), retos al poder de la nación-Estado (globalización), programas reguladores que muchos aceptan en principio, pero que no gustan en la práctica (en áreas tales como la protección ambiental y los derechos civiles) y la aparición de movimientos políticos hostiles al gobierno y a los gastos del gobierno (no hay voluntad de pagarlos). Si pudiésemos estar de acuerdo sobre qué clase de políticas gubernamentales queremos - cómo queremos tratar los derechos a las prestaciones, la escasez de recursos, el problema de la competencia global-, entonces gran parte del resto estaría resuelto. La ‘buena gente de la que habla el informe del NPR podría dirigir el sistema de una forma que la mayoría aceptarían —con o sin las reformas del NPR-, aunque ciertamente ayudada por mejores técnicas de gestión y contando con la atención en los temas de gestión de aquellos que diseñan y supervisan las políticas.

Un gobierno mejor que costase menos sería bienvenido y las reformas de la gestión pueden ayudar. Pero por el momento no está claro que las reformas de la gestión del NPR en y por sí mismas son la respuesta, incluso donde son adoptadas. Son los recortes en programas y los beneficios los que producen mayores ahorros. $\mathrm{O}$, alternativamente, un vigoroso crecimiento económico que hace innecesarios los recortes o amortigua su impacto. Los ciudadanos americanos dicen que quieren gobiernos más pequeños, pero también están considerados como los mejor administrados la mayoría de los programas de mayor gasto nacional del gobierno, en particular los grandes como la Seguridad Social y los seguros médicos del Estado. La clave para el país está en decidir, a través de sus líderes, qué quiere y lo qué está dispuesto a pagar, aun cuando las reformas de gestión son también importantes. En otras palabras, las reformas de la gestión (juiciosamente aplicadas) pueden ayudar a solucionar 
algunos problemas, tales como la excesiva regulación, pero las decisiones sobre lo que debería o no hacer el gobiemo son, en último término, una labor de los funcionarios elegidos en democracia. Uno puede reducir los costes de personal (concretamente, los beneficios) con la subcontratación, por ejemplo, pero el gran recorte proviene de la reducción o eliminación del programa en sí. Decidir lo que es básico y lo que no es necesario, en resumen, es fundamentalmente una cuestión politica y no de gestión.

Otro punto muy importante es el relativo a las nociones de planificación estratégica que son fundamentales en la familia de las reformas del New Public Management. Algunos cuestionan la conveniencia de la planificación estratégica en el entorno contemporáneo. Por ejemplo, Jonathan Boston y June Pallot, dos agudos estudiosos de las reformas en Nueva Zelanda, contrastan la planificación estratégica y el pensamiento estratégico:

De cara a los acelerados cambios (tales como la revolución de la información, la globalización y la democratización en comunidades y lugares de trabajo), la planificación estratégica se ha visto atacada porque la predicción de las discontinuidades es virtualmente imposible y las predicciones son casi invariablemente enróneas y su sistema analíico de cálculo pierde la información más ssutil y los matices importantes. A diferencia de la planificación estratégica, el pensamiento estratégico está basado en la creatividad, la intuición y el aprendizaje organizativo; las estrategias a menudo no pueden desarrollarse sobre un esquema y concebirse perfectamente, sino que deben ser libres de aparecer en cualquier momento y lugar en una organización ${ }^{75}$.

No podemos evaluar aquí las diferencias entre estas dos visiones de la gestión, pero sería irónico que los gobiernos adoptasen la concepción pasada de recortar del sector privado en su impetu por gestionar de una manera más parecida al sector público.

Al evaluar la iniciativa de la reinvención también se deben tener en cuenta las difíciles cuestiones relativas a la función del gobierno. Como KeTTL senala: .Por mucho que los reformadores incorporasen modelos del sector privado a las operaciones del gobierno, el gobierno no es ni será una empresa. En una democracia, su labor fundamental es perseguir el interés público. Promueve valores cruciales como la imparcialidad, la justicia, la equidad, el derecho a un procedimiento justo. El gobierno existe ... precisamente porque el mercado privado y la gestión empresarial realizan un pésimo trabajo en perseguir objetivos tales como éstos que van más allá de la eficiencia ${ }^{l}$.

KETTL cree que la "gestión por resultados" es completamente compatible con, y de hecho favorece, que el gobierno persiga el interés público ${ }^{75}$. Arrojando luz sobre qué están logrando los programas del gobierno, se ayudaría a mejorar la gestión y tam- bién se ayudaría a los ciudadanos a hacer evaluaciones de los resultados del gobierno. Los críticos tienden a recalcar que ven una incompatibilidad entre el enfoque hacia el cliente del NPR y los objetivos del gobierno de los Estados Unidos. James $C_{A}$ RROLL expresa este punto elocuentemente:

En lugar de los propósitos y valores proclamados en el Preámbulo de la Constitución como objetivos de un gobierno constitucional - una unión más perfecta, la defensa común, la tranquilidad nacional, la justicia, el bienestar general, los beneficios de la libertad- [el NPR] los sustituye por los valores de una sociedad de consumo -la satisfacción del consumidor. Convierte al gobierno en un instrumento de consumo de servicios, ignorando el papel del gobierno en la resolución de conflictos, en el establecimiento de objetivos nacionales, en el control del uso de la fuerza en la sociedad, en la inversión en el fururo de la nación, en la consecución de los valores constitucionales y los objetivos políticos, lo cual tiene poco o nada que ver con el servicio o la satisfacción de los consumidores ${ }^{76}$.

En el fondo del debate sobre el New Public Management está el malestar sobre su énfasis en la eficiencia y la satisfacción del consumidor (como opuesto a ciudadano). Parte del problema es la potencial incompatibilidad entre ambos. Pero, como las citas de KeTtr y CaRROLl dejan claro, también existe tensión entre lograr ciertos fines políticos, entre los que podríamos pensar en la eficiencia política y la eficiencia en la gestión. Cuanto más se acepta la noción de que el gobierno existe para promover la imparcialidad, la justicia, la equidad y el derecho a un proceso justo, más importante es especificar dónde se deberían enfatizar las técnicas de NPR/NPM y dónde deberían ser retiradas al fondo del armario.

Finalmente, como James Q. WILSON dice, no se puede reinventar con éxito el gobierno de forma seria sin repensar qué debe hacer el gobierno." Gran parte de la iniciativa de la reinvención incide en las técnicas de gestión, muchas de ellas inportantes y útiles, pero la primera cuestión es qué quieren los ciudadanos que el sector público haga y sólo entonces cuál sería la mejor forma de conseguir estos objetivos.

\section{Agradecimientos}

Expresamos nuestro agradeciniento a la Brookings Institution y al personal del Governmental Studies Program, especialmente a Robert Katzmann y a Thomas Mann, por dotarnos de una beca, despachos y financiación durante el mes que pasamos en Washington cuando se escribió la mayor parte de este artículo. Joel Aerbach reconoce con agradecimiento el apoyo 
del Academic Senate y del Center for American Politics and Public Policy en UCLA y la ayuda en la investigación concedida por Wendy Hunter. Bert Rockman agradece a Paul Mullen su ayuda.
Traducción de Gemma Sisculz: Romovi:.

- Universidad de California, Los Ángeles.

" Universidad de Pitrsburgh.

1 Este aniculo se extrae del Capírulo 7 (.Reinventing Government.) de nuestro libro que aparecerá próximamente, In the Wed of Politics: Three Decades of the U.S. Federal Executive (Washington, D.C.: The Brookings Institution, próximla aparición). Se publica aqui con el permiso de Brookings Instirution. Una versión ligeramente diferente se presentó en el Grupo de Trabajo sobre Los Politicos, los Burócratas y la Reforma Instirucional, de la Sesión Conjunta de los Grupos de Trabajo del Consorcio Europeo para la Investigación Política, Mannheim, Alemania, 26-31 de marzo de 1999, y la 57 lomada Anual de la Asociación de Ciencia Politica del Medio Oeste, Chicago, Illinois, EE.UU., 15-17 de abril de 1999.

' Al Gons:, Crealing A Govenunent Thal Works Better and Costs Less. El prólogo al informe pone de manifiesto que hacer que el gobierno funcione mejor y cuesie menos son las dos •misiones gemelas. del NPR. Véase p. xxiii.

"La cita es de Donald F. Kl:Irt, Reintenting Gonermment: A Fifth-Year Report Card, Un informe del Centro para la Gestión Pública de la Brookings Institution, CPM 98-1, septiembre de 1998, p. 5. Véase también Jolı M. KAMs:Nsky, Role of the Reinventing Government' Movement in Federal Management Reformm. Public Administration Review, vol. 56, no 3 (majo/junio, 1996), p. 251.

- GoRs:, Creating A Governmen That Works Better and Costs Less, pp. xxxii-xxxiii.

; lbid., p.129.

$\checkmark$ Ibid., p. xxrriii.

7 Wrivecderg/pumal

' Gonl, Creating A Government That Works Better and Costs Less, pp. xanviii.

${ }^{9}$ Christopher Hoon, .De-Sir Humphreyfying the Westminster Model of Bureaucracy: A Nerr Sryle of Governance?, Goternance, vol. 3, no. 2 (abril 1990), p. 207.

"Malcom Holuts y David SHAil), Management Reform: Some Practitioner Perspectives on the Past Ten Years, Goternance, vol. 8, no. 4 (ocrubre 1995), pp. 551.51 .

"Naomi Calbs: A New Generation of Budget Reform., p. 252, en Guy Ptaters y Donald J. Sivoll:, Taking Stock: Assessing Public Sector Reforms (Montreal: McGillQueens Universily Press, 1998)

1. Véase el prólogo al Final Report of the Lean State Advisory Commitiee (1997) al Gobierno Federal alemán, elaborado por su Presidente, el Profesor Rupen Scholz. Dice, p. 3, •La idea de un 'Estado mínimo' o la necesidad de modernizar el Estado, ha llegado a ser actualmente la habladuria de la ciudad'. El apojo a este objetivo es casi universal, aunque, desde luego, las exhaustas reservas del Estado, así como una participación del sector público que es demasiado elevada, incluso a la vista de una rendencia descendente, han ajudado de muchas formas a ser más conscientes : crear opciones en esta área.

${ }^{13}$ B. Guy Pmi:Rs, The Future of Goneming (Lantence: University of Kansas Press, 1996), pp. 1,13. Véase también, entre otros, Donald J. Sivoll:, Globalization and Covernance, en B. G. Plitz:Rs y' D. I. Sivoll:, eds., Govemance in a Clsanging Enturon. ment (Montreal; MCGill/Queens Universit): Press, 1995, yt Jolin Kamlixsky del staff del NPR, Role of the 'Reinventing Government' Movement in Federal Nanagement Reformi, p. 248.

If Richard Rosficknice, -The Rise of the Virtual Siate, Foreign Affairs, vol. 75, no. 4 (julio/agosto 1996), p. 60.

is Donald F. Kl:lit, ·The Global Revolution in Public Management: Driving Themes, Missing Links., Joumal of Policy Analysis and Management, vol. 16, no. 3 (1997), p. 447. Cursiva en el original.

16 Ibid., 447.

${ }^{17}$ Ibid., p. 448. Cursiva en el original.
18 Ibid., p. 448.

19 Ibid., p. 449.

20 James D. Carkoul, The Rhetoric of Reform and Political Reality in the National Performance Revien', Public Administration Revieul, vol. 55, no. 3 (majo/-junio 1995), p. 30 ?.

"1 Ki:lin, Reinuenting Gonemunenl: A Fifib-Year Report Card, pp. 5-6.

$n$ GoRl:, Creating A Got:entment That Works Better and Costs Less, p. 96.

2. Ibid., p. 116.

34 lbid., p. 117.

$s$ lbid., p. 145 .

loid., p. 160 .

"Informe del National Performance Revien de 1995, Common Sense Gonernment, (9/7/95) Apéndice C: Nen: Recommendations by Agency, pp. 1-24.

${ }^{38}$ Se puede indagar más sobre este tema en el capítulo 8 de nuestro próximo libro, In The Web of Politics: Three Decades of The U.S. Federal Executive. A modo de explicación, deberiamos señalar que la Fase III de la reinvención buscó, en palabras de Kl:Tr, pasar de los anteriores recortes de plantilla a un énfasis en los resultados., aunque a veces desafortunadamente era sobre la consecución de los resultados Icomo lograr comunidades seguras] sobre lo que los gobiernos federales jugaron un papel muy pequeño en su producción. Véase Kl:Trn, Reinuenting Govermment: $A$ Fifit)-Year Report Card, pp. 5-6.

* Donald F. Kl:T'tl, Reimlenting Gonemment?' Appraising the National Perfonmance Retient, A Report of the Brookings Institutions Center for Public Management, CPM 94-2, 19 de agosto de 1994, pp. v-vi.

"Ibid., p.v.

"Por ejemplo, véase Warren E. LlinkY, NASA Learns That Faster and Cheaper Isnt Always So', Nern York Times, 15 de septiembre de 1998, pp. B9, B13. LEarY describe el fracaso de la NASA en gestionar contratos para dos satélites que iban a construirse y lanzarse en dos años con un coste de 60 millones de dólares cada uno. En el sistema diseñado para poner en práctica la políica de la NASA de sllás rápido, mejor y más barato, se les dio a los contratistas mucha libertad de acción para alcanzar los resultados, con un conjunto de incentivos $y$ desincentivos de gestión puestos en práctica para garantizar su cumplimiento e impedir excesos en los cosies. El concepto parecia bueno sobre el papel, pero la falia de seguimiento por parte de la NASA su. puso que la agencia no siempre era consciente de muchos de los problemas que los contratistas estaban teniendo hasta que fue demasiado tarde.

"Herben Kiur:Mn, Red Tape: Its Origins, Uses and Abuses (Washington: The Brookings Institulion, 1977), concretamente el capítulo 2.

"James Q. W/LSON, Reinventing Public Administration., PS: Political Science \& Politics, vol. 27, no. 4 (diciembre de 1994), p. 670.

"Peler Aucols, •Administrative Reform in Public Management: Paradigms, Principles, Paradoxes and Pendulums, Gotemance, vol. 3, no. 2 (abril 1990), p. 132; véase también, H. George Frlidt:kICKSON, .Comparing the Reinventing Government Movement with the Nen Public Administmation, Public Administration Rerietu, vol. 56, no. 3 (majo/junio 1996), p.266.

is Linda de LtON y Roben B. DE.NIIARDT, The Political Theory of Reinvention, un ar. liculo preparado para la Reunión Anual de la Asociación Americana de Ciencia Politica de 1997, Washington, DC, 27-31 de agosto de 1997, pp. 8, 10.

${ }^{36}$ KAMENSSKY, Role of the Reinventing Government' Movement in Federal Management Reformi, p. 252.

$\because$ GoRl:, Creating a Gonermment Thal Works Better and Costs Less, p. xcxiji.

36 Ibid., pp. 107-111. 
WILsos, Reinventing Public Administration, p. 671.

- Ronald C. AlOE, The Reinventing Govemment Exercise: Alisinterpreting the Pro blem, Misjudging the Consequences, Public Administation Revier1, rol. 34 , no. 2 (majo/junio 1994), p. 119.

i) Fredericksos, Comparing the Reinventing Govemument Mtovement nith the New Public Management, p. 268.

i? Paul C. LuGr, Tbickening Goiemment: Federal Hierarcby and tbe Diffusion of ACcomntability (Washington: The Brookings Institution, 1995).

"3. Brina SANGER, revisión de Thickening Gorernment, Jourmal of Policy Analysis and Management, vol. 15, no. 1 (inviemo de 1996), p. 126.

${ }^{4}$ Véase The Report of the National Commission on the Public Service, Lendersbip for America: Rebuilding the Public Senrice (Washington, 1989), pp. 16-19. La sección se tirula Make more room near the top for canter execulives.

i) Kt: $\mathrm{n}$, The Global Revolution in Public Management, cit., p. 457.

${ }^{16}$ United Staies General Accounting Office, The Goternment perfonnance and Results Act: 1997 Governmenturide Implementalion Win Be Unet:en, GAO/GGD-97-109, p. 96.

1 National Performance Review, Savings: Brief Explontion of Savings Estimates and had the): nere Derived, NFR Savings 1997, mmw nprgov/libran/papers/Dkgrd/explair:himl, pp. 1-3.

*5 KETrn, ·The Global Revolution in Public Management?, p. 456.

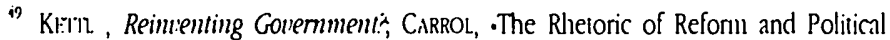
Reality: in the National Performance Revien:, pp. 307, 309.

"Frl:DI:RICNSON, Comparing the Reinventing Government Movement with the New Public Administration, p. 269.

"Gore, Creating a Gol:ermment That Works Better and Costs Less, pp. 109-114.

': Kl:Tll, Reimlenting Goternment: A Fifbt-Year Report Card, p. 5.

"Frederick M. KuIst:R y Virginia A. MCMUURTRY, Government Performance and Results Act: Implications for Congressional Orersight, CRS Report for Congress (Congressional Research Service, Biblioteca del Congreso), 97-382-GOV, 24 de marzo de 1997, p.1.

; Government Performance And Resulis Act de 1993 (S. 20, Enrolled Bill Sent to President), Sec. 4, enmienda al Capítulo 11 del tífulo 31, Código de los Estados Unidos, Sec. 1115, Planes de Actuación.

is Ibid., Sec. 1115

* KiISI:R y MCMUKIRY, Government Performance and Resulis Act, cit., p. 15.

i7 lbid., p. 5.

is United States General Accounting Office, Managing for Results: Agencies'Annual Performance Plans Can Help Address Strategic Planning Challenges., GAO/GGD-98. 44 (enero de 1998); United Siates General Accounting Office, Managing for Results: The Stanutory' Framenork for Performance-Based Management and Accountabilij: $\cdot$, GAO/GGD/AIMD-98-52 (enero 1998).

i) United States General Accouting Office, Managing for Results: The Stantory Framenork for Performance-Based Management and Accouniability', p.19.
6) United States General Accounting Office, 1 lanaging for Results: Agencies Annual Perfomlance Plans Can Help Address Sirategic Planning Challenges, pp. 3-4.

at United Siates General Accounting Office, The Govemment Perfonilance and Resulis An: 1997 Govemmentride Implementation Will Be Uneven, GAO/GGD-97-109, pp. 56 .

62 El Congreso no aceptó la idea de que se establecieran objetivos para las agencias. Sin embargo, el Senador Williant Roth (Rep. Del.), un campeón del GPRA, no opinaba lo mismo. El escribió los siguientes puntos de visia adicionales sobre la ley en el informe del Comité del Senado sobre Asuntos Gubemamentales: En el contexto de la legislación, a las agencias federales se las exigiria que desarrollasen objetivos medibles para sus programas. Creo que deberiamos dar un paso más allá y también pedir al Congreso que juegue un papel directo en el estableciniento de al menos algunos de esios objelivos. El Congreso crea y financia programas, asi que deberia dar alguna indicación sobre lo que espera que ellos consigan ... El Congreso liene la obligación de decir a los contribujenies americanos cuáles son los resultados que se preienden con el dinero que gastamos y esia exigencia deberia incluirse en la legislación. Véase El Senado de los Estados Unidos, Comité sobre Asuntos Gubernamentales, Government Performance and Results Aa, S. Report 103-58, 16 de junio de 1993. (Washington, D.C.: U.S. Govermment Printing Office), p. 57. Gmacias a Robert D. Behn por hacernos llegar esta cita $y$ a Susan McGrath de la Brooking Institution Library por facilitamos una copia del informe del comité.

os CClinton Adminsiration Fails Accountability Revien-, Freedom Works: Home Page of the Office of the House Mayority Leader (The Results Acl: It's the Law, El Informe de Noviembre de 1997), hup://freedom.housegov/resulis/finalrepor/releaseasp, p.1.

Gt Anne Liurtist, -The Resulis Act: Playing Chicken, GonErec, A Service of Gorem.

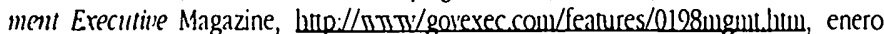
de 1998, p. 1.

(s) Ibid., p. 2.

" KuIST:R : NCMURTRY, . Government Perfonmance and Results Act: Implicacions for Congressional Oversightr, p. 8.

G) Ibid., p. 13.

(s) Ibid., p. 15

(6) United States General Accounting Office, The Government Performance and Results Act: 1997 Governmenmide Implementation Will Be Uneven, p. 6.

Ibid., p. 6.

"lbid., p. 7.

1" Para una discusión sobre estos temas, véase el capirulo 1 de $h$ the Web of Politics.

"Jonathan Bosion y June Pil.or, binking Strategy and Pperformance: Developments in the Nen: Zealand Public Secior, Journal of Policy Analysis and Manage. ment, vol. 16, no. 3 (1997), p. 386

"Donanld F. Kl:Tlt, The Global Revolution in Public Management: Driving Themes, Missing Links, p. 459 .

I Ibid., p. 460.

" Iames D. Carkoll, .The Rhetoric of Reform and Political Reality in the National Performance Reviens, p. 309.

$\pi$. lames Q. W/LSON, .Reinventing Public Adminsitmtion?, p. 672 


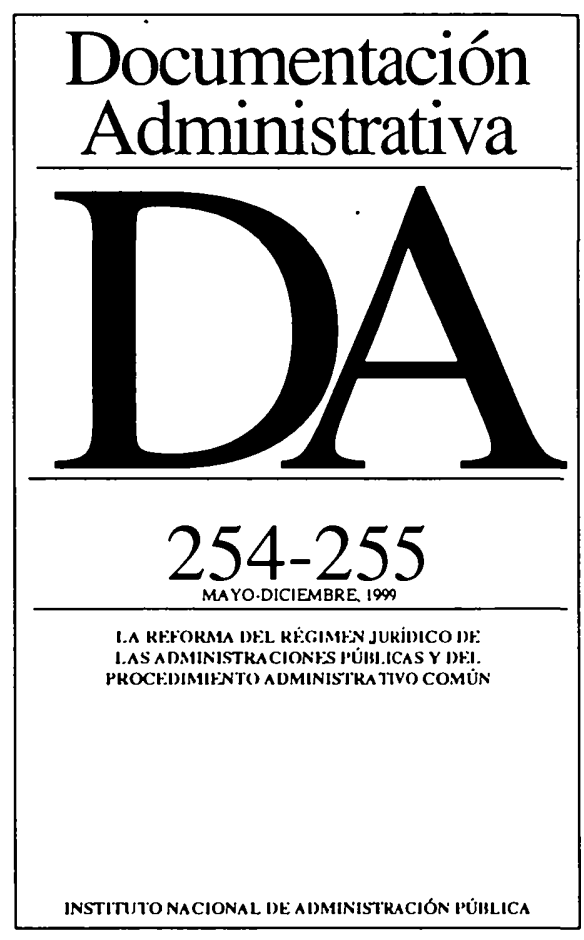

Publicación cuatrimestral del Instituto Nacional de Administración Pública, especializada en Derecho Administrativo y Organización y Gestión de las Administraciones Públicas.

Aparecida en 1958, la característica más destacada de su actual etapa es la dedicación monográfica de cada número a una cuestión especifica, que se analiza desde diferentes puntos de vista por acreditadas firmas de España y otros países.

\section{CONSEJO DE REDACCIÓN}

Presidente: Enrique Álvarez Conde. Vocales: José Javier Abad Pérez, Óscar Alzaga Villamil, Manuel Aragón Reyes, Enrique Argullol Murgadas, Gaspar Ariño Ortiz, Miguel Beltrán Villalva, Andrés de Blas Guerrero, José Manuel Castells Arteche, Ínigo Cavero Lataillade, Rafael Gómez-Ferrer Morant, Francisco González Navarro, Jesús González Pérez, Luis Morell Ocaña, Sebastián Martín-Retortillo Baquer, José Ramón Parada Vázquez, Eduardo Roca Roca, Ángel Rodríguez García, Juan Alfonso Santamaría Pastor, Gumersindo Trujillo Fernández.

\section{Director \\ Luciano Parejo Alfonso \\ Secretario \\ Jesús Prieto de Pedro}

\section{Edita:}

MINISTERIO DE ADMINISTRACIONES PÚBLICAS Instituto Nacional de Adminisiración Pública

\section{Suscripciones y Administración:}

Instituto Nacional de Adininistración Pública Publicaciones Periódicas Atocha, 106. 28012 Madrid

Teléfono: 3493104

\section{Precios:}

